\title{
Christian Buson
}

\section{L'écologie, une science confisquée?}

\author{
Conférence donnée à Florac, en Lozère, le 6 août 2018, en hommage à Claude Monnier \\ (1916-2018)
}

Recibido: 18 novembro 2018 / Aceptado: 22 novembro 2018

(C) Universidade de Santiago de Compostela 2018

\begin{abstract}
Résumé Après avoir présenté les effets de l'irruption de l'écologisme dans les préoccupations de la société, nous abordons une critique de l'écologie en tant que discipline scientifique. Nous relevons que nombre de postulats et d'hypothèses s'enchaînent sans être réellement vérifiés, au point que les certitudes sont minces et qu'une attitude plus circonspecte et précise s'appuyant sur des observations et mesures sur le terrain sont indispensables. Les erreurs au prétexte de l'écologie sont innombrables et exceptionnellement désignées comme telles. Les recommandations et contraintes qui en sont déduites reposent sur des approximations la plupart du temps fort discutables. Les travers de l'écologie politique sont également décelables dans les articles scientifiques. La nécessité d'une direction de recherche renouvelée et plus objective est abordée, de façon à ce que l'écologie basée sur les preuves se développe enfin, à l'écart des a priori et des convictions. II est important que les décisions soient retenues en tenant compte des résultats acquis sur l'environnement réel.
\end{abstract}

«En relisant l'ouvrage, je suis surpris et peiné par le caractère modéré et courtois du ton. Je regrette de ne pas avoir su parler de quelques outrecuidantes escroqueries, fumisteries et fourberies intellectuelles de notre époque avec moins de retenue.» Romain Gary ; Pour Sganarelle, 1965.

\author{
Christian Buson \\ Docteur en agronomie \\ Institut Scientifique et Technique de l'Environnement \\ Liffré 35340 France \\ Tfn : 0299685151 - Fax : 0299041025 \\ Email: Christian.buson@ges-sa.fr
}

Resumen Presentamos una crítica de la ecología como disciplina científica. Observamos que numerosos postulados e hipótesis se encadenan sin ser realmente verificados, hasta el punto que las certezas son escasas. Es indispensable adoptar una actitud de mayor circunspección y precisión, apoyándose en las observaciones y mediciones realizadas sobre el terreno. Los errores cometidos en pretexto de la ecología son innumerables y en raras ocasiones reconocidos como tales. Las recomendaciones y limitaciones que así se deducen están basadas, la mayor parte del tiempo, en aproximaciones muy discutibles. Los defectos de la ecología política son reconocibles incluso en artículos científicos. Se aborda la necesidad de adoptar una dirección de investigación renovada y más objetiva, para desarrollar por fin la ecología basada en pruebas, alejada de los a priori y las convicciones. Es fundamental que las decisiones sean adoptadas sobre la base de resultados adquiridos en el entorno real.

«Releyendo la obra, me sorprendo y apeno por el carácter moderado y cortés del tono. Me arrepiento de no haber hablado de ciertas estafas arrogantes, engaños y manipulaciones intelectuales de nuestra época con menor contención.» Romain Gary; Pour Sganarelle, 1965.

\section{Qu'est-ce que l'écologie?}

Ernst Haeckel avait défini en 1866, l'écologie comme «la science des relations des organismes vivants avec le monde environnant ". A l'origine, l'écologie est une science qui cherche à étudier les interactions entre les organismes vivants et le milieu. C'était une approche nouvelle, synthèse de nombreuses sciences préexistantes. La question centrale porte sur cette synthèse et la hiérarchie entre toutes les disciplines de base, pour aborder correctement chacun des sujets. Les préoccupations écologiques ont surtout fait irruption à partir des années 1970. Elles ont complexifié de nombreux domaines. 


\section{L'écologisme : une prétention à guider nos vies}

Cette approche a été jugée novatrice, si ce n'est révolutionnaire et a inspiré de nombreux mouvements politiques : ainsi est apparu ce qu'il est convenu d'appeler l'«écologie politique», autrement dit «l'écologisme».

Plusieurs traits caractérisent ces mouvements :

- La dramatisation et les alertes sur les dangers majeurs et les catastrophes irréversibles qui menaceraient la faune, la flore, les mers, les continents, la planète et l'espèce humaine, du fait des activités économiques jugées inconsidérées ;

- La recherche du pouvoir et du monopole de l'information;

- La priorité accordée à l'environnement, à la biodiversité et à la planète, et le discrédit permanent des activités humaines, surtout celles des pays développés ;

- L'usage d'une phraséologie radicale, voire révolutionnaire, préconisant au final un renforcement du corsetage réglementaire, débouchant sur des interdits, des taxes et des contrôles : le bien ne serait que ce qui a été écrit dans les textes réglementaires ; ceci explique l'activisme des lobbies sur les textes réglementaires nationaux et de l'union européenne ; les nécessaires corrections et adaptations des textes réglementaires, à la lumière des connaissances, sont particulièrement laborieuses à obtenir ;

- Implicitement, l'«écologiquement correct» ne pourrait provenir que de la puissance publique et non des acteurs privés et des citoyens ; cet arsenal législatif à la complexité croissante, réconforte et occupe nombre de juristes, alors que les citoyens, les agriculteurs et les entrepreneurs ne comprennent plus ce qu'on attend d'eux ;

- La remise en cause et l'ignorance de la production, des filières progressivement mises en place par les sociétés humaines, et au final de l'économie de marché ;

- La limitation de la liberté, des déplacements et de la démocratie, sous prétexte des prétendues «lois de l'écologie», dont on ignore encore à peu près tout (1).

Qui a énoncé «les lois de l'écologie»? Sont-elles disponibles et vérifiées au point que toutes nos politiques devraient s'en inspirer? Evidemment nous n'en sommes qu'aux balbutiements, et notre premier devoir consiste à ne pas les prendre pour acquises a priori, même si certains s'en font les porte-voix.

Comment un arsenal réglementaire s'appuyant sur des bases aussi fragiles, discutables et fluctuantes saurait-il encadrer utilement nos actions? Comment éviter de déboucher comme souvent sur des blocages, des taxes, de la paperasserie, des contrôles, des obligations à tous propos?

Il est évident qu'apprécier le plus tôt possible l'impact des interventions humaines ou d'un aménagement est un préalable indispensable et que nombre de progrès ont été accomplis en quelques décennies dans ce domaine, notamment dans les pays dits occidentaux, et en particulier en France depuis la mise en place en 1974 des études d'impact précédant les aménagements importants. II est possible d'éviter, de réduire et de compenser les nuisances prévisibles dès la conception des projets. C'est ce qui est développé pour les établissements soumis à la légistation des Installation classées pour la protection de l'environnement (ICPE) ou pour les Autorisations Environnementales, désormais.

Mais, jusqu'ici il faut bien constater que les préoccupations écologistes ont conduit à une succession d'erreurs, de théories invérifiées et de contre-sens majeurs et, parfois, ces erreurs intrinsèques des concepts écologistes ont été «fixées» par la réglementation, qu'il est ensuite difficile de faire évoluer, à plus forte raison avec le principe de non régressivité du droit de l'environnement selon lequel la protection de l'environnement, assurée par les dispositions législatives et réglementaires relatives à l'environnement, ne peut faire l'objet que d'une "amélioration constante» (cf. Falque 2017). Comme s'il était aisé de définir une «amélioration» et «sa constance».

Au fil des années, l'écologie politique nous a montré combien ses divisions étaient nombreuses, incessantes et renouvelées ; il n'y a aucune unanimité sur l'analyse de la situation, ni sur les solutions, face à chacune des questions soulevées. Au final, les propositions de l'écologisme en termes politiques débouchent sur des propositions assez indigentes et partiales ; celles-ci n'ont d'ailleurs reçu qu'une approbation fort limitée dans l'opinion, comme en témoignent les résultats électoraux des formations politiques se réclamant de l'écologie.

Comme pour la psychanalyse, il faut s'interroger si l'écologie politique n'est pas «une maladie qui se prend pour son remède» : poser des questions, parfois intéressantes, ne suffit pas à formuler des réponses pertinentes et acceptables par le plus grand nombre.

L'écologie politique et ses propagandistes nous ont habitués à leur discours grandiloquents, emprunts de catastrophisme, d'irréversibilité et d'irrémédiabilité, de menaces toujours plus terribles pour la santé et l'environnement, toujours plus d'épidémies meurtrières, de perturbations gravissimes, de pertes drastiques de la biodiversité par ailleurs en grande partie inconnue, de risque d'extinction de l'espèce humaine au XXlème siècle (annoncée comme certaine par notre ancien ministre de l'écologie, Nicolas Hulot, dans «le syndrome du Titanic»). Il faut également se remémorer le film d'Al Gore "une vérité qui dérange» dont les prophéties spectaculaires se sont avérées fausses. Toutes ces prévisions n'engagent que ceux qui les écoutent et ne pourront jamais être vérifiées ; les lanceurs d'alerte qui les professent le font «par précaution» et se savent être hors d'atteinte de toute critique ; il est vrai que des prévisions sur le long terme sont autant gratuites qu'invérifiables.

(1) Par exemple, une illustration récente : dans un interview donné à Libération le 29 juillet 2018, M. François-Marie Bréon, chercheur en climatologie et directeur adjoint du laboratoire des sciences du climat et de l'environnement (LSCE), énonce les mesures radicales à mettre en œuvre dans la lutte contre le réchauffement climatique. II affirme: «On peut dire que la lutte contre le changement climatique est contraire aux libertés individuelles et donc sans doute avec la démocratie.» http://www.liberation.fr/planete/2018/07/29/francoismarie-breon-la-lutte-pour-le-climat-est-contraire-aux-libertes-individuelles_1669641 «La propagande réchauffiste» selon l'expression de Rémy Prud'homme (2015) nous prépare-t-elle à une nouvelle forme de dictature? 
Les politiciens qui s'emparent des thèmes écologistes tentent vainement de rejustifier l'économie administrée ; ils auraient réponse sur tous les sujets : la production et l'utilisation de l'énergie, nos modes de vie, notre production agricole et industrielle, le nombre d'enfants dans les familles, notre consommation, nos modes de transport... ; La liste des préconisations à prétexte écologique, qui sont attentatoires à notre liberté est assurément longue et infiniment extensible.

Faut-il croire ces prophéties alarmistes? Le mot «croire» est d'ailleurs approprié. Selon le Pr. Marian Apfelbaum, l'homme qui est omnivore, devrait s'efforcer de «croire» parce que sa survie en dépend. L'influence des religions décline, les drames gigantesques générés par les idéologies totalitaires du XXème siècle sont reconnus. Dès lors, de nouvelles idéologies de substitution peuvent prospérer : il faut s'interroger si cela ne constitue pas, la fonction sociétale de l'écologisme.

Par bien des aspects, l'écologisme fonctionne comme une religion à laquelle il serait devenu impératif de croire (cf. Crichton 2003). L'écologie, constitue-t-elle cette nouvelle religion au service d'un nouvel ordre mondial, comme l'avait prédit Luc Ferry (1992)?

De nombreux auteurs nous ont déjà avertis des risques consistant à prendre pour acquises les peurs développées par les mouvements écologistes : Gérard Bramoulé, Bernard Oudin, Claude Allègre, Max Falque, Jean de Kervasdoué, Luc Ferry, , Christian Gérondeau, François Gervais, Vaklav Klaus, Bjørn Lomborg, Claude Monnier, Rémy Prud'homme, Didier Raoult, Benoit Rittaud, Haroun Tazieff,... Pour n'en citer que quelques-uns...

\section{L’écologie, science des écologues?}

Une distinction entre cette écologie politique volontiers radicale et démagogique, avec une nouvelle science des écologues est apparue nécessaire. Les écologues ont développé leurs activités scientifiques, en dehors des écologistes, acteurs ou militants politiques.

Signalons en préambule que fort peu de scientifiques interviennent pour démentir ou nuancer les propos inconsidérés développés sous prétexte de la «science écologiste». Le sort médiaticopolitique réservé à ceux qui osent tenter la contradiction, comme Claude Allègre ou Bjørn Lomborg, explique d'ailleurs probablement la prudente réserve qu'ils adoptent.

Fondamentalement, une science est une discipline dans laquelle les théories et les hypothèses sont vérifiées; dans le cas où elles sont infirmées, elles sont abandonnées et la théorie doit être renouvelée avec de nouvelles orientations.

Dans le cas de l'écologie scientifique, la vérification des hypothèses n'est pas systématiquement menée. De sorte que, de la formulation des hypothèses à leur répétition, nous assistons ensuite à l'adoption d'affirmations sans preuves. L'enchevêtrement des hypothèses invérifiées aboutit à des fondations précaires et, au final, à un édifice incertain et fragile : par contre, l'angoisse entretenue des catastrophes et bouleversements imminents est porteuse dans les médias et en tant que produit politique ; l'appel aux changements radicaux et urgents se trouve ainsi facilement validé, répété, amplifié.

La théorie du climax, émise en 1916 par Frédéric E. Clements, qui décrète que les écosystèmes non perturbés par l'homme tendraient vers un état d'équilibre, stade ultime et supposé idéal de leur évolution, a intéressé nombre d'écologues ; cette théorie relève clairement de l'utopie et n'a jamais pu être démontrée ; ce concept du climax entretient une vision statique de l'équilibre de la nature, alors que nombre d'expériences montrent par exemple qu'une forêt non entretenue se transforme en quelques décennies en une jungle impénétrable et inhospitalière.

De même, l'hypothèse Gaia de James Lovelock qui considère la planète terre comme un être vivant malade qu'il faudrait soigner, n'a jamais pu être vérifiée.

Une théorie, fût-elle partagée par un grand nombre de scientifiques de diverses disciplines, reste une théorie. Pour autant, les paradigmes en cours dans les milieux scientifiques ne peuvent tenir lieu de démonstrations.

Face à chacune des affirmations, il faut exiger la preuve que celles-ci sont démontrées. C'est la révolution qui a eu lieu en médecine, avec l'«evidence-based medicine» (la médecine par la preuve et les faits EBM). «La médecine fondée sur les preuves consiste à utiliser de manière rigoureuse, explicite et judicieuse les preuves actuelles les plus pertinentes lors de la prise de décisions concernant les soins à prodiguer à chaque patient».

Force est de constater que l' «evidence-based Ecology» reste à mettre en place.

Comme en psychologie ou en sociologie, nous assistons en permanence à une succession d'hypothèses qui s'enchaînent, sans que les prémisses et le discours ne soient correctement vérifiés. La notion même de «démonstration» est contournée. Par bien des aspects, l'écologie scientifique qui se veut également globale, affirme largement au-delà de ses capacités à démontrer : l'hubris est manifeste et produit des dogmes, qui sont autant de "prêts à penser», mais qui peuvent se révéler des «colosses aux pieds d'argile».

Souvent faute de connaissances, d'observations précises, de reculs suffisants dans les constats et mesures, établir des conclusions est délicat. Au lieu d'en faire le constat, les laboratoires ont recours assez systématiquement à la modélisation. Mais une modélisation qui tente une simulation de l'évolution d'un système complexe, ne prouve absolument rien. II convient au minimum que le modèle soit correctement validé par une comparaison entre les simulations obtenues et les mesures effectuées sur le terrain.

Notons qu'une modélisation permet la production de nombreux articles dans des revues scientifiques, mais ne constitue jamais une confirmation de l'exactitude du modèle. Tout modèle repose sur un schéma conceptuel et des hypothèses simplificatrices ; le modèle ne peut fournir des résultats absolus. II doit être vérifié a posteriori par des 
mesures de terrain correctement interprétées, étape indispensable bien souvent négligée ou éludée. En outre, comme le souligne le Pr. Didier Raoult, la modélisation du vivant est pratiquement impossible.

En écologie, les interactions entre les nombreux êtres vivants et la multitude des paramètres rentrant en jeu, rendent difficiles les généralisations à partir des observations effectuées. La mise en place d'expériences, ne faisant varier qu'un paramètre et «toutes choses égales par ailleurs» est quasiment impossible. Les expériences en aveugle ou en double aveugle ne sont qu'exceptionnelles, de sorte que nombre d'écrits relevant de l'écologie scientifique ne démontrent rien et se contentent de répéter les théories admises dans leur discipline. Le paradigme est ainsi cultivé.

En outre l'écologie, science jeune, ne dispose qu'exceptionnellement de données comparables à des époques antérieures. Ceci limite considérablement les possibilités d'apprécier correctement la moindre évolution. Or nous constatons que dans les articles relevant de l'écologie, les conclusions émises éludent cette absence de références historiques et prennent pour certains les résultats observés et les tendances d'évolution hâtivement imaginées.

Parallèlement, l'intérêt de ces thèmes pour le marketing des laboratoires de recherche et la carrière des chercheurs est indéniable : les experts concluent souvent sur la justification du renforcement des recherches sur les sujets qu'ils ont eux-mêmes imaginés. Cette position de «juge et partie» n'est qu'exceptionnellement remise en cause. Si l'urgence est assimilée, l'affectation de fonds pour financer les recherches afférentes devient alors prioritaire, ce qui avantage les laboratoires concernés.

Une autre tendance fréquente dans ces disciplines consiste à ce que nous pouvons résumer par «faire parler la biologie ou l'écologie». L'exemple de la formule banale : «la nature a horreur du vide», illustre cette idée. Or la biologie ne pense assurément pas. Et il faut absolument se départir de cette inclination. Le développement végétal ou animal se produit lorsque les conditions sont favorables et que la compétition favorise les espèces concernées.

Dans ce contexte, le public est souvent «obligé de croire», sans possibilité de vérification, ni de réfutation.

Quelques exemples d'erreurs manifestes de l'écologie :

-Les erreurs dans l'histoire des sciences sont innombrables et l'écologie n'y échappe pas ; nous en rappelons ici quelques-unes :

- Le refus par les géologues des théories de Wegener sur la "dérive des continents»; 40 ans de retard sur la compréhension des mécanismes géologiques majeurs : orogénèse, volcanisme, tremblements de terre, tsunamis et autres «catastrophes naturelles»...,

- Le refus des théories de Pasteur sur l'absence de génération spontanée par l'Académie de Médecine,

- Le refus des gastro-entérologues de reconnaître les causes microbiennes des ulcères de l'estomac et en particulier du germe helicobacter pilori, alors qu'ils soutenaient que les ulcères étaient causés par le stress, les aliments épicés ou un excès d'acidité... La plupart des ulcères est désormais traitée par antibiotiques ; en 2005, un prix Nobel de médecine a été attribué à $B$. J. Marshall et J. Robin Warren, pour leur découverte et leur ténacité face à l'adversité...

Nous développons ci-après plus complètement quelques sujets relatifs aux erreurs de l'écologie dite scientifique et des conséquences qui en ont été trop hâtivement tirées.

\section{Les cas des nitrates vis-à-vis de la santé et de l'environnement}

En ce qui concerne la santé, il est incohérent qu'une norme à $50 \mathrm{mg}$ de nitrate par litre d'eau de boisson ait pu perdurer, alors que nombre de légumes présentent naturellement des teneurs 10 à 100 fois supérieures, en raison de la physiologie des plantes dont les racines prélèvent les nitrates dans la solution du sol. Les nitrates sont évidemment les mêmes dans l'eau et dans nos aliments et ne peuvent avoir que les mêmes propriétés après ingestion dans l'organisme. De plus, les recommandations des nutritionnistes de consommer des légumes en abondance sont généralisées dans le monde entier, ce qui implique donc de consommer davantage d'ion nitrate.

Nous savons désormais que les nitrates et les nitrites sont les précurseurs de l'oxyde nitrique (NO), molécule dont la découverte du rôle a été couronnée par le prix Nobel de médecine en 1998 : l'oxyde nitrique régule la circulation sanguine tout au long de l'existence et prévient et soulage les troubles cardiaques et les maladies dites liées à l'âge. La consommation de nitrates est bénéfique pour la santé, sans limite de dose. En outre, il s'avère que la voie Nitrate-Nitrite-Oxyde Nitrique assure un mécanisme de défense naturelle vis à vis des agents potentiellement pathogènes (cf. travaux de Nigel Benjamin). Ces "NOx» sont autant de molécules aux effets bénéfiques pour notre santé. L'acharnement réglementaires pour les éliminer de notre environnement et les normes sur les émissions de NOx sont d'autant moins compréhensibles.

De même le rôle des nitrates sur l'environnement a fait l'objet de contre-sens majeurs concernant l'eutrophisation que ce soit en eau douce ou en eau marine côtière.

Pour les proliférations algales d'eau douce, ce sont des algues microscopiques (cyanobactéries) fixatrices d'azote atmosphérique qui en sont responsables. Ces proliférations peuvent intervenir quel que soit le taux de nitrate dans l'eau, dès lors que les autres conditions sont réunies (température, lumière et taux de phosphore disponible dans l'eau...).

Concernant les proliférations massives d'ulves sur quelques rares plages en Bretagne, l'hypothèse du lien avec les flux totaux ou printaniers d'azote terrigène n'a jamais pu être confirmée par des mesures. Les ulves échouent sur les baies favorables du fait de la courantologie et de la nature de l'estran. Les rivières débouchant sur ces baies propices n'apportent que des flux d'azote limités très inférieurs à ceux 
charriés par de nombreux autres fleuves côtiers, comme la Vilaine par exemple, sans qu'aucun désordre sur la croissance des macro-algues n'ait été observé. Les ulves ne constituent nullement ces «éponges à nitrate» qui nous sont décrites dans les médias.

Les préconisations des experts de l'Ifremer pour «lutter contre» ces marées vertes - il faut noter le langage guerrier adopté à ce propos -, sont issues de modélisations invérifiées faute d'observations suffisantes de l'écologie de l'ulve dans le contexte armoricain. Les travaux de Joël Kopp insistant sur le rôle essentiel des bigorneaux, principaux consommateurs des ulves, ont été ignorés par ses successeurs. Ces bigorneaux ont d'ailleurs connu une forte régression ces dernières décennies, sans que celle-ci ait été correctement étudiée.

La stratégie préconisée en France consiste à réduire les quantités d'azote, au point de créer une faim d'azote dans le milieu marin, et ainsi permettre une limitation de la croissance des algues. Mais il est bien évident que cet objectif est aussi absurde qu'inaccessible, les sources d'azote marin étant nombreuses et importantes, sans oublier l'impact écologique que cette carence en azote générerait sur l'écologie marine, si l'objectif recherché était atteint.

Tout a été publié et explicité que ce soit sur les eaux douces et les eaux marines côtières, notamment dans les articles de Guy Barroin et de David Schindler.

Or ce sujet des nitrates et de ses conséquences sur la santé et l'environnement constitue un axe essentiel de la politique environnementale de l'Union Européenne et de la France depuis plusieurs décennies et la parution de la directive Nitrates de 1991. A la lumière des connaissances avérées, ce sujet devrait être définitivement abandonné, vue sa vacuité. Mais les structures d'alerte, de recherche, d'accompagnement, de contrôles et de sanctions rechignent à la mise à plat du sujet, par crainte probable de perdre une partie de leur supposée «raison d'être».

\section{La directive cadre sur l'eau}

Un autre exemple de l'écologie approximative traduite en terme réglementaire nous est fourni par la Directive Cadre sur l'Eau de 2000 dans laquelle l'union européenne prévoyait le retour rapide au «bon état écologique» des masses d'eau, objectif auquel tout lecteur ne peut qu'adhérer a priori. Mais la définition du bon état écologique des masses d'eau est précisée plus loin : l'état dans lequel seraient les masses d'eau sans l'influence de l'activité humaine et économique. Où sont les scientifiques qui ont cautionné ou validé cette réglementation? Quel scientifique est capable de caractériser objectivement les masses d'eau en question? Plus fondamentalement, quel écologue a contesté cette définition irréaliste? Qui a conservé une "aquathèque» pour préciser la qualité de ces eaux originelles? Résultat : incertitudes, dispersion des règles entre les états membres, les régions, distorsions de concurrence, etc...

\section{La théorie du réchauffement ou dérèglement climatique}

Celle-ci est également intéressante à considérer : comme si la température moyenne du globe pouvait avoir un sens physique, comme si un écart de $1^{\circ}$ Celsius sur un siècle et demi pouvait être significatif, compte-tenu des imprécisions des mesures de températures, surtout au XIXème siècle, et après des corrections incertaines effectuées sur les températures à proximité des zones urbanisées, comme si de tels écarts (infimes en réalité) ne pouvaient entraîner que des bouleversements colossalement négatifs pour les êtres vivant et les populations, et comme si aucune adaptation des espèces n'était concevable... II est fort probable que dans quelques décennies la désinformation et la fièvre collective qui accompagnent la promotion des actions urgentes et formidablement coûteuses pour lutter contre le réchauffement climatique, feront l'objet d'études savantes pour tenter d'expliquer comment pareille folie a pu s'emparer des opinions et des meilleurs esprits, comme autrefois «l'utopie socialiste» selon l'expression de Jean-François Revel. Voir le site des climato-réalistes https ://www.climato-realistes.fr/ et, entre autres, l'intervention de Vaklav Klaus https://www.climato-realistes.fr/contresommet-climato-realiste-vaclav-klaus/

La notion même de «dérèglement climatique» est un contre-sens implicite, le climat présentant partout, en tous lieux et en toutes périodes, y compris géologiques, des variations importantes ne suivant aucune «règle».

Mais les affirmations ne sont qu'exceptionnellement assorties de preuves de leur véracité et ne reposent que sur leur répétition. Signalons au passage que les articles publiés dans de nombreuses spécialités qui prennent désormais pour acquis dans leur préambule ou leur conclusion ces postulats invérifiés du «dérèglement climatique», sans préciser comment leurs travaux les confirmeraient, entretiennent l'illusion dans l'opinion de leur justesse «scientifiquement établie». C'est le cas par exemple quand un prix Nobel d'économie comme Jean Tirole, tente de calculer les conséquences économiques du réchauffement climatique, alors qu'il n'en vérifie pas la réalité. Les prévisions de Nicholas Stern en 2006 avaient déjà relevé des mêmes procédés. Peu d'intervenants et de très rares écologues ont critiqué ce biais pourtant manifeste.

\section{L'agriculture, l'écologie et la biodiversité}

L'agriculture moderne fait l'objet d'un discrédit en France, autant généralisé qu'injustifié(2), y compris dans de nombreux travaux et articles dits scientifiques : pourtant les gains de rendements moyens en France par exemple ont augmenté de près d'un quintal par ha et par an pendant 40 ans. Nous sommes capables avec les connaissances agronomiques connues et éprouvées du XXlème siècle de nourrir et de maintenir en bonne santé 12 milliards d'humains, avec des menus variés et agréables, intégrant des produits d'origine animale. L'espérance de vie, qui a fortement progressé ces dernières décennies, confirme

(2) Sylvie Brunel a publié en 2017 un exceptionnel « Plaidoyer pour nos agriculteurs ». Beaucoup trop d'agronomes ne s'autorisent pas un tel courage aujourd'hui. 
l'amélioration de la qualité de notre alimentation qui est devenue accessible quasiment à toute la population mondiale.

II faut bien considérer que les activités agricoles ou forestières se sont développées, sans perturbations environnementales majeures et sans que les biologistes ne perdent leurs sujets d'études et de recherche sur la biodiversité dans les espaces naturels et modifiés par l'homme. Les «ravages» et les «méfaits» présentés comme irréversibles qu'entraîneraient les activités agricoles et d'élevage sont fortement exagérés, même s'ils sont affirmés constamment. De tels propos devraient discréditer leurs auteurs, mais nous constatons que le plus grand nombre les laisse dire ou les répète, y compris dans le personnel scientifique. Comme si ce «clin d'œil» adressé à l'opinion, était un signal de bienveillante compréhension des scientifiques, quelle que soit la part d'inexactitude qu'il entretient...

De retour de Madagascar, où nous avons mené une étude pédologique pour un futur domaine sur lequel une école d'agriculture pourrait s'implanter à proximité de Mananjary, nous avons constaté par exemple, que les sols, par leur organisation, leur structure, leur profondeur, leur porosité... présentaient une importante aptitude à tous types de production, mais que les réserves minérales y étaient infimes.

Le recours à une fertilisation soutenue et ajustée est un préalable incontournable à toute mise en valeur des sols. Par ailleurs, malgré l'occupation des sols, proche de la friche, et un climat tropical favorable aux activités biologiques, la biodiversité est particulièrement ténue : pas ou peu d'insectes, d'oiseaux, de mammifères... ; nous avons là un exemple manifeste que, dans un contexte d'agriculture largement extensive, voire d'absence d'agriculture proprement dite, la biodiversité peut être réduite à sa plus simple expression, contredisant ainsi l'affirmation que la perte de la biodiversité serait due à l'agriculture, hâtivement qualifiée d'intensive, pour la parer a priori de tous les maux.

La coexistence d'une agriculture productive et d'une biodiversité soutenue est facile à observer, partout dans le monde ; mais ce sont des caricatures catastrophistes qui sont régulièrement rapportées, générant une désinformation de l'opinion, y compris dans les milieux scientifiques.

Dans «La Nature en débat : idées reçues sur la biodiversité», Christian Lévêque critique cette tendance souvent observée : «il est regrettable que des chiffres discutables concernant l'érosion de la biodiversité soient utilisés en permanence pour sensibiliser l'opinion. Les estimations quantitatives restent le plus souvent du domaine de la spéculation. On peut rappeler à ceux qui l'auraient oublié, que Paul Ehrlich, inventeur de la bombe «P» (la bombe démographique) et gourou de la biodiversité, annonçait au début des années 1980 que 250 000 espèces disparaissaient chaque année, et que la moitié de la biodiversité aurait disparu en l'an 2000. Ce qui pourrait prêter à sourire si beaucoup de scientifiques n'avaient cautionné ces élucubrations sans broncher ! Selon un autre gourou, le biologiste Edward Wilson, la moitié des espèces actuellement présentes sur Terre pourrait avoir disparu d'ici un siècle. On se donne rendez-vous?... L'affirmation selon laquelle le taux d'extinction serait, de nos jours, 100 à 1000 fois supérieur à celui des formes fossiles, est devenus un dogme. On la sort à tous propos, mais on cite rarement la source. ... Une lecture attentive de ce travail [une publication de 1995 parue dans la revue Science, dont Stuart Pimm est un des co-auteurs] relève néanmoins qu'il y a beaucoup d'approximations et d'hypothèses non vérifiées aussi bien pour l'érosion actuelle que pour le calcul du taux d'extinction naturelle que l'on peut qualifier de bruit de fond... Le calcul du taux d'extinction qualifiée de naturelle, se heurte au fait que nous ignorons les formes fossiles qui n'ont pas laissé de traces. ॥

II faut également reconnaître, concernant la perte de la biodiversité souvent mise en avant pour critiquer nos sociétés modernes, que nous ne connaissons qu'une faible partie des espèces animales et végétales vivant sur la planète, mais que des espèces nouvelles se créent en permanence ; de plus l'examen des fossiles établit que le nombre d'espèces ayant disparu à travers les âges géologiques est considérable. D'innombrables extinctions massives de grande ampleur ont parsemé l'histoire géologique, et pas seulement lors de la transition entre le crétacé et le Tertiaire (K-T), à laquelle la disparition des dinosaures est attribuée, et cela sans que l'activité humaine puisse évidemment être mise en cause (cf. travaux de J. Sepkoski et D. Raup).

Le scientifique, comme tout un chacun, sait que sur certains sujets qu'il maîtrise, l'exagération est de mise, mais il a tendance à croire que sur nombre d'autres sujets, si la suspicion perdure, c'est qu'elle relève probablement d'une réalité démontrée dans d'autres domaines que ceux qu'il maîtrise.

Ainsi la disparition des abeilles, qui est au moins en partie due à l'insuffisance des traitements contre le parasite Varroa destructor, ou la disparition annoncée comme drastique des insectes, des oiseaux, ou de la Mulette perlière, sont-elles souvent admises sans preuve, ni surtout sans mesures comparatives fiables à travers les âges.

A l'inverse, face à la forte recolonisation des territoires par le loup, observée en France, nous constatons une bienveillance a priori des milieux scientifiques (lycophiles) et une édulcoration de leur rôle de prédateurs sur les troupeaux. De tels biais nuisent évidemment à l'objectivité et à la qualité des travaux établis par les experts. Au final, il faut s'interroger si cela n'explique pas la perte de crédit des experts scientifiques dans l'opinion, sans parler sur les réseaux sociaux.

\section{L'agroécologie, l'agriculture biologique et la permaculture}

Nous assistons à la mise en avant des vertus supposées de formes nouvelles d'agricultures supposées plus respectueuses de l'environnement de la biodiversité et des équilibres écologiques de la planète, qui sont désormais regroupées sous le terme d' «agroécologie». Le discours est plaisant à entendre, forme de marketing New Age.

L'agroécologie : une discipline nouvelle (?) à la consonnance prometteuse mais qui n'a jamais montré de résultats probants sur le terrain. C'est le nouveau qualificatif englobant l'agriculture biologique (ou organique des 
anglo-saxons), la biodynamie, l'agroforesterie, la permaculture...

Opposer la chimie à la biologie est absurde : aucune espèce ne peut vivre sans les éléments chimiques, de l'eau, de l'air, des sols... La biologie "fonctionne» dans toutes les parcelles agricoles quel que soit le type d'agriculture pratiqué. L'agriculture biologique, biodynamique, l'agroforesterie, la permaculture n'ont pas le monopole des réalités biologiques qu'utilisent toutes les agricultures.

L'agriculture biologique rejette les lois de la chimie agricole, mises à jour au XIXème siècle, après les travaux de Liebig, chimiste prussien, sur l'indispensable fertilisation pour compenser les exportations minérales effectuées par les cultures. Ces notions seraient jugées «trop anciennes et trop proches du complexe militaro-industriel» qui aurait «recyclé ses surplus dans l'agriculture» (sic !). Il est utile de visionner la surprenante vidéo dans laquelle un ancien Président Directeur Général de l'INRA apporte sa caution à ces mouvements militants et à ce type d'affirmations péremptoires :

http://www.institut-environnement.fr/index.php?option=com content\&view $=$ article\& $i d=112 \&$ Itemid $=29 \& 6 b 6 d 4$ e $1 \mathrm{c} 63$ 988a0adbec4940a17c9332=fc3b7a1ebd1608a54d93e1924 d462a70

Jamais les produits issus de cette agriculture ne se sont avérés meilleurs ni pour la santé, ni pour l'environnement, ni pour leur goût, malgré les discours répétitifs entretenus sur ces sujets. Comment par exemple, une agriculture qui ne quantifie pas ses apports de fertilisants, mais se contente d'en valider l'usage au moyen d'une liste restrictive, peut-elle affirmer qu'elle polluerait moins? Du fumier en surdosage provoquera la même surfertilisation qu'un engrais minéral, qu'il soit accepté ou non en agriculture biologique.

La «nouvelle tendance» souvent mise en avant de nos jours, concerne la «permaculture», contraction d'agriculture permanente, comme si la production agricole ou maraîchère actuelle n'était pas durable... «Elle n'est pas une méthode figée mais un «mode d'action» qui prend en considération la biodiversité de chaque écosystème»... Comprendre les bases de cette agriculture est une gageure : il s'agirait de favoriser l'optimum de toutes les espèces animales et végétales, ce qui permettrait également une fourniture soutenue de légumes et de fruits pour l'alimentation humaine. Cette idée de «l'optimum simultané» de toutes les espèces est une vue de l'esprit, ignorant les compétitions que mènent les espèces entre elles (la fameuse «struggle for life») et les nombreux parasites et maladies de toutes sortes dont les jardiniers ont toujours tenté de s'affranchir par leurs observations et leur travail.

Il est certain que ce type d'agriculture ou de maraîchage ne produira pas grand-chose de fiable ni de commercialisable durablement, si ce n'est des stages payants, pour bien persuader les vagues successives de candidats tentés par cette aventure.

Les jardiniers, les maraîchers, les agriculteurs n'avaient pas trouvé comment faire depuis des siècles; les permaculteurs nous affirment qu'ils y parviennent. S'il suffisait d'y penser et de le déclarer (cf. «oasis en tout lieu» du mouvement des colibris, inspiré de Pierre Rabhi)!

Les leçons de morale souvent délivrées aux pays en développement sont par ailleurs indécentes : quand les populations sortent de la misère et de la faim, elles aspirent à bénéficier d'un mode de vie proche de celui des pays occidentaux ; il serait fort malvenu de les en dissuader, du haut d'une nouvelle morale écologiste.

Pour aider les populations qui ont faim, inutile de promouvoir des modes de productions supposées novatrices, relevant de «l'agroécologie», qui n'ont, jusqu'ici, jamais fait leurs preuves et dont les bases conceptuelles accumulent les contre-vérités. Les techniques de productions et de conservations classiques correctement conduites, permettront de répondre efficacement aux attentes et à la santé de tous les consommateurs, en nombre croissant sur la planète, et cela sans perturbations préjudiciables de l'environnement.

\section{L'irruption de la mode Végan}

Cette orientation devenue omniprésente prend pour acquises les thèses de l'animalisme, du «droit des animaux» et de «l'antispécisme»: la question centrale porterait sur la légitimité du droit que l'espèce humaine s'arrogerait de domestiquer d'autres espèces. Eric Denécé, en 2016, a détaillé les comportements de ces activistes dans son ouvrage donné en référence.

Comme sur de nombreux sujets, les protagonistes s'autorisent à formuler "ce que pense la biologie » (espèces animales et végétales, voire les minéraux) et s'en font son porte-parole. Des titres d'associations comme «Sources et Rivières du limousin», "Eau et Rivières de Bretagne», ou encore Bretagne Vivante (Société pour l'Étude et la Protection de la Nature en Bretagne (SEPNB)), entretiennent l'illusion que c'est l'objet qui s'exprime, la voix de la nature révélée. L'écologie profonde dispose d'un slogan radical : "penser comme une montagne», avec des démarches violentes et illégales que leur discours «en rupture» légitimerait. Nous assistons à longueur d'émissions, d'articles, d'ouvrages et de documentaires à des propos profondément erronés, mais laissés sans réponse.

Incontestablement, l'espèce humaine est omnivore ; nous sommes issus de populations ayant pratiqué la chasse et la cueillette pendant des millénaires. L'agriculture et l'élevage ont permis de spécialiser, d'améliorer les productions et de les rendre facilement disponibles pour les populations.

Les protéines végétales ne contiennent pas tous les acides aminés nécessaires, contrairement aux protéines animales. Du point de vue nutritionnel, les produits d'origine animale présentent des avantages que le végétal ne peut compenser ; ceci est particulièrement bien développé notamment dans les ouvrages du Dr. Jean-Marie Bourre. Le refus de consommation de tous les produits d'origine animale (lait, œuf, viande, poisson, miel...) est une position idéologique infondée et quasi sectaire. 
S'il est certain que tous les animaux sont des êtres sensibles, il est patent que dans toutes les chaînes alimentaires, des êtres sensibles consomment d'autres êtres sensibles, et ce, sans état d'âme. C'est vrai du lion qui consomme la gazelle, du loup qui consomme un agneau, de la pieuvre qui consomme un crabe, de l'oiseau qui consomme un ver ou un insecte...

S'il faut se préoccuper du bien-être animal, comme tout éleveur digne de ce nom le fait depuis toujours, le bien-être des humains doit constituer une priorité, y compris dans leurs travaux et pour leur alimentation. Face à cela, nous constatons le désarroi des producteurs et des acteurs des filières, «de la fourche à la fourchette» : comment répondre au catastrophisme et à l'avalanche de réglementations? Faudrait-il supprimer les filières d'élevage et de transformation des produits animaux? Et dans l'hypothèse où l'élevage de ruminants serait empêché, que deviendraient les millions d'hectares de pâturages sur la planète? La consommation de l'herbe des prairies par des animaux ne constitue-t-elle pas une formidable adaptation écologique?

Au final, nous apprécierions d'entendre les réponses des végans, sur comment faire face aux divers parasites, microbes, virus, souris, rats, mouches, moustiques, charançons et autres insectes, etc... qui sont assurément indésirables.

\section{La sanctuarisation des zones humides}

Les zones humides font l'objet d'une préservation renforcée inscrite dans le code de l'environnement. II s'agit de «terrains, exploités ou non, habituellement inondés ou gorgés d'eau douce, salée ou saumâtre de façon permanente ou temporaire ; la végétation, quand elle existe, y est dominée par des plantes hygrophiles pendant au moins une partie de l'année». (Art. L.211-1 du Code de l'Environnement).

En fait dans tous les paysages, certains sols peuvent présenter une circulation de l'eau ralentie. De tous temps l'agriculture a cherché à se prémunir des excès d'eau et à protéger les cultures, les animaux et les infrastructures. II s'agissait de travaux d'assainissement, car en outre ces milieux pouvaient être insalubres pour les populations alentours. Pour cela, les sociétés humaines ont créé un parcellaire, avec des fossés d'évacuation des eaux excédentaires. De plus les parcelles les plus fréquemment engorgées en eau ont fait l'objet de travaux de drainage : implantation d'un réseau de canalisations enterrées en poterie, puis en plastique, suivant un plan incliné facilitant l'écoulement des eaux. II ne s'agit pas d'assécher les sols, les cultures ayant toujours besoin d'eau pour se développer, mais d'améliorer le ressuyage après les épisodes pluvieux pour éviter la stagnation de l'eau dans toute la porosité du sol, ce qui limite l'activité racinaire et le développement végétal.

A travers les âges, de profondes modifications des conditions initiales ont été conçues et mises en œuvre par les générations successives, pour limiter les effets de ces excès d'eau. C'est le cas par exemple de l'aménagement des polders ou de la Camargue...

II convient en outre de mentionner que les zones de marais émettent naturellement des quantités importantes de méthane et que leur aménagement et les drainages permettent la réduction de la teneur en gaz dit à effet de serre dans l'atmosphère.

Le retour à l'état de nature préalable à tous ces aménagements n'est ni souhaitable, ni une amélioration environnementale.

\section{Les limitations du droit de propriété}

Désormais, au motif que les milieux humides présenteraient une biodiversité importante, les actions possibles pour améliorer l'hydraulique, voire pour entretenir les réseaux existants sont particulièrement limitées. Ceci rend les aménagements de ces zones quasiment impossibles. Les terres ne présentent plus d'intérêt et leur valeur marchande baisse drastiquement.

Ceci constitue un exemple de la limitation du droit de propriété. II en existe d'autres : les milieux étant réputés participer d'une fonction écologique d'intérêt majeur, les obligations s'imposant aux propriétaires s'accroissent. Les limitations du droit de propriété au prétexte écologique sont nombreuses.

Le cas de l'interdiction de la chasse, par exemple, qui a été imposée dans certains parcs nationaux et régionaux, a entraîné des dégâts considérables sur les massifs forestiers notamment le bois d'Altefage en Lozère, ce qui a été lourdement sanctionné ensuite par la justice, au terme de longues procédures (25 ans). Ceci a été raconté dans le détail par Monsieur Renaud de Laubespin. http ://www.accac.eu/decouverte_aigoual/Docs/De_Laubespin_ Renaud.PDF

De même, lors de la constitution de périmètres dits de protection des captages d'eau destinée à la consommation humaine, une série d'obligations s'impose aux propriétaires et aux exploitants sans que la justification de ces contraintes ne soit établie, ni que leur respect garantisse le maintien d'une bonne qualité de l'eau. Comme le fait remarquer l'association France Captages qui regroupe des propriétaires et des agriculteurs concernés par ces périmètres de protection, la bonne qualité actuelle des eaux résulte du travail des agriculteurs et ce de longue date. II est difficile d'admettre que l'adoption de contraintes stéréotypées puisse améliorer de quelque façon que ce soit la qualité des eaux. Cette eau de bonne qualité est un produit de leur travail, pour lequel les agriculteurs ne sont aujourd'hui pas rémunérés.

D'une manière certaine, l'écologie égrène des interdits, des contraintes réglementaires. L'administration est considérée comme le garant autant central qu'incontournable du respect des écosystèmes et de leur fonctionnement harmonieux. Les obligations et les interdictions faites aux propriétaires sont sans cesse accrues. Cette réglementation, qui ne prend jamais en compte la capacité 
et l'intérêt des propriétaires à préserver les fonctionnements écologiques de leurs domaines fonciers, commet là un contre-sens manifeste. Nous constatons que fort peu d'écologues interviennent pour discuter ce parti pris aux conséquences fâcheuses.

\section{Pour conclure}

Les erreurs concernant l'écologie sont innombrables et nous n'en avons illustré dans cet article qu'un nombre forcément limité. Les surprises sont importantes, dès que l'on cherche à approfondir chacun des sujets.

«Le plus grand défi qui se pose à l'humanité c'est le devoir de distinguer la réalité de l'imaginaire, et la vérité de la propagande. Percevoir la vérité a toujours été un défi pour l'humanité, mais à l'ère de l'information (c'est à dire, désormais, à l'ère de la désinformation), cela prend une "urgence et une importance cruciale.» Michael Crichton, 2003. C'est normalement la mission affectée à la science. Nous constatons que la science écologique, qui est une science jeune, et qui de ce fait manque de références et de comparaisons historiques, ne clarifie pas suffisamment cette nécessaire distinction.

Une science doit fondamentalement vérifier ses hypothèses de façon objective et ne pas hésiter à rejeter toute erreur ou approximation incertaine. L'écologie scientifique doit encore faire des progrès dans ce domaine pour justifier son statut de science et que celle-ci ne soit plus «confisquée», notamment par des lobbies.

La place de l'homme et des activités humaines ne doit pas être considérée a priori comme une série de contraintes et d'actions uniquement néfastes, mais comme une réalité qu'il convient de d'intégrer aux réflexions et orientations, et si possible avec bienveillance. L'écologie et la biodiversité, avec l'homme, et non "contre l'homme», s'imposent.

Les ouvrages de Christian Lévêque : «L'écologie est-elle encore scientifique?», "La nature en débat», ou "La biodiversité avec ou sans l'homme?», abordent ces questions essentielles qui sont autant de critiques de la production scientifique actuelle dans ces domaines où les convergences avec l'écologisme sont encore souvent manifestes. Une direction de recherches dans le domaine de l'écologie reste à mettre en place.

Comme pour toutes réglementations, celles à connotations écologiques doivent faire l'objet périodiquement de vérifications de leur bien fondé et d'adaptations aux connaissances nouvelles ; le cas échéant certains textes doivent pouvoir être abrogés et un minimum de nouveaux textes efficaces établis. L'intérêt et la préservation des droits de propriétés et de la liberté qui y est attachée doivent être reconnus.

L'humilité doit être la règle face à nos incompréhensions actuelles de l'ensemble des mécanismes biologiques et écologiques ; en particulier, il convient de se garder de «faire parler la biologie», comme le sous-entendent nombre d'écologistes, mais aussi trop d'écologues. La notion de «pollution» doit être réservée aux émissions qui perturbent effectivement la biologie par leur concentration, leur devenir et leur nocivité.

Les écrits de l'économiste Julian Simon en 1998, avaient amené à Bjørn Lomborg, «l'écologiste sceptique», à entreprendre des vérifications et à constater au final que la quasi-totalité des prévisions écologiques étaient erronées et inutilement alarmistes : "Les conditions matérielles de la vie continueront indéfiniment d'aller de mieux en mieux, pour la plupart des gens, dans la plupart des pays, la plupart du temps. Dans un siècle ou deux, toutes les nations et la plupart de l'humanité seront à l'équivalent ou au-dessus du niveau de vie occidental actuel. J'imagine pour autant que la plupart des gens continueront de penser et de proclamer que les conditions de vie s'aggravent.» Or, c'est exactement ce que nous constatons actuellement dans l'enseignement dispensé de la maternelle à l'enseignement supérieur ou dans les alertes médiatiques qui se répètent en boucle ; les milieux scientifiques relevant de l'écologie ne s'attachent pas suffisamment à nous éclairer sur ce qui relève de l'intox ou de la réalité. La peur entretenue au sujet des "perturbateurs endocriniens», sans qu'on sache correctement en préciser la définition, ni le risque réel que leur présence entraînerait, illustre par exemple la confusion développée dans les médias, notamment par les membres d'un laboratoire du Muséum National d'Histoire Naturelle.

Aussi, est-il temps, selon nous, de nous tourner vers les sciences de l'environnement et de la santé. II convient de baser fermement nos décisions et nos actions sur ces sciences ; il s'agit de l'environnement réel, vérifié ; il est utile d'établir une correcte hiérarchie permettant de fixer des priorités, et de «négliger le négligeable».

Un état des lieux objectif de la réalité environnementale sur le terrain est un préalable systématique avant de prendre de «bonnes décisions». Cela évitera de voir le château de cartes s'effondrer ensuite.

De nombreuses découvertes et quantité de solutions innovantes pourront encore contribuer à l'amélioration des conditions de vie humaine, et dans un environnement harmonieux. Ne sous-estimons pas nos capacités d'adaptation. Le pire est loin d'être certain.

En paraphrasant Michaël Crichton, nous avons besoin d'une science écologique renouvelée, avec de nouveaux objectifs et de nouveaux processus de validation, avec plus de scientifiques sur le terrain, dans notre environnement réel et moins de gens devant des écrans d'ordinateurs qui commentent des simulations issues de modèles incertains.

Pour en savoir plus,

\section{Bibliographie}

Académie des technologies 2015, Le méthane d'où vient-il et quel est son impact sur le climat? 170 pages

http://academie-technologies-prod.s3.amazonaws.com/201 5/01/06/10/55/21/635/rapport_methane_version_web.pdf 
Allègre C. 2010, L'imposture climatique ou la fausse écologie. Editions Plon, 295 pages.

Barroin G. 2003, Gestion des risques Santé et Environnement: le cas des nitrates. Phosphore, azote et prolifération des végétaux aquatiques. Assises internationales Envirobio. Editions de l'ISTES et

Courrier de l'environnement de l'INRA février 2003. Disponible sur: http://www.inra.fr/dpenv/barroc48.htm

Barroin G. 2004, Phosphore, Azote, Carbone... Du facteur limitant au facteur de maîtrise. Courrier de l'environnement de l'INRA $n^{\circ}$ 52, pp 1 à 25. Disponible sur: http://www.inra.fr/dpenv/pdf/barroc52.pdf

Bourre J.-M., Buson C., L'hirondel J.-L., 2011 Nitrates, nitrites, oxyde nitrique (NO): nouvelles perspectives pour la santé? Médecine \& Nutrition $47 n^{\circ} 2$, pp. 43-50, (c) EDP Sciences.

Bourre J.-M. 2003, La diététique du cerveau, la nouvelle donne, Editions Odile Jacob. 302 pages.

Bourre J.-M. 2003, Bien manger: vrais et faux dangers. Editions Odile Jacob. 333 pages.

Bourre J.-M. 2003, Le Lait: vrais et faux dangers, Editions Odile Jacob. 367 pages.

Bramoulé G. 1991, La peste verte. Editions Les Belles lettres.

Brunel S. 2017, Plaidoyer pour nos agriculteurs. II faudra demain nourrir le monde... Editions BuchetCastel. 127 pages

Bryan N. S. 2010, Food, Nutrition and the Nitric Oxide Pathway. DEStech Publications. 218 pages.

Buson C. 2005, «Retour «écologique» sur la question des nitrates», Recursos Rurais (2005) Vol1 n¹: 39-49 IBADER: Instituto de Biodiversidade Agraria e Desenvolvemento Rural ISSN 1885-5547. Disponible sur http://www.ibader.org/archivos/docs/Recursos\%20Rurais \%2001-05.pdf

Buson C., Buson B., Mauger V., Agrelo Yañez M. Y. 2012, Développement et échouages d'Ulves en

Bretagne: pour une souhaitable réorientation des recherches; Recursos Rurais $n^{\circ}$ 8: 49-55. ISSN

1885-5547. http://www.usc.es/revistas/index.php/rr Revue Officielle de I'IBADER (Institut de Biodiversité Agricole et Développement Rural), USC (Université de Santiago de Compostela)

Buson C. Apfelbaum M., Bardinet J.-P., Beslu P., Gérondeau C., Houdebine L. M., Julien J.-L., L'hirondel J.-L., Monnier C., Proust J.F., Veyres C., Voron H. 2016, Réponse à l'écologisme, comment la connaissance permet de réfuter les peurs entretenues. Editions l'Harmattan. 354 pages.

Crichton, M. 2003, L'écologisme en tant que $r$ e I i g i o n http://www.forumphyto.fr/images/pdf/DocusPublics/2010/0 309crichtonecoreligion.pdf https://www.cs.cmu.edu/ kw/ crichton.html
Crichton M. 2007, Etat d'urgence. Editions Robert Laffont. 646 pages.

Crichton M. et Preston R. 2011, Micro. Livre posthume. Editions Robert Laffont. 476 pages.

Denécé E., Abou Assi J. 2016, Ecoterrorisme. Altermondialisme, écologie, animalisme: de la contestation à la violence, Taillandier.

Falque M. 2016, Propos écologiquement incorrects, Editions Libréchange. Vol 1: 238 pages et Vol 2: 277 pages.

Falque M., Hernandez-Zakine C., Peignot B., 2017 le principe de non-régression saisi par le droit de l'environnement, revue du droit rural $n^{\circ} 457$ Novembre 2017, pp. 12-15.

Ferry L. 1992, Le Nouvel Ordre Ecologique, L'arbre, l'animal et l'homme. Grasset. 280 pages.

Gave C. 2016, Sire, surtout ne faîtes rien ! Vous nous avez déjà assez aidés. Editions Jean-Cyrille Godefroy. 247 pages.

Gérondeau C. ,2012. Ecologie, la fin. Vingt ans de décisions ruineuses. Editions du Toucan. 299 p.

Gervais F. 2013, L'innocence du carbone, L'effet de serre en question, contre les idées reçues. Albin Michel. 317 pages. de Kervasdoué J. 2014, Ils ont perdu la raison. Editions Robert Laffont. 229 pages.

de Kervasdoué J. 2016, Ils croient que la nature est bonne, Ecologie, agriculture, alimentation: pour arrêter de dire n'importe quoi et de croire n'importe qui. Editions Robert Laffont. 178 pages.

Klaus V. 2009, Planète Bleue en Péril Vert, Qu'est-ce qui est en danger aujourd'hui: le Climat Ou la Liberté ? Iref.

Kopp J., 1977 Etude du phénomène des marées vertes en Bretagne; rapport de synthèse. ISTPM, 102 pages de Lavoisier A. 1789, Traité élémentaire de chimie, FB Editions. 139 pages.

Lévêque C. 2013. L'écologie est-elle encore scientifique? Editions Quae. 230 pages.

Lévêque C. 2017 La biodiversité avec ou sans l'homme ? Réflexions d'un écologue sur la protection de la nature en France, Editions Quae. 127 pages.

Lévêque C. 2011, La Nature en débat: Idées reçues sur la biodiversité. Editions Le cavalier Bleu. 172 pages.

L'hirondel J., L'hirondel J.-L. 2004, Les nitrates et l'homme: Toxiques, inoffensifs ou bénéfiques? Editions de l'Institut de l'Environnement, 256 pages.

Lomborg B. 2002, The skeptical environmentalist, Measuring the real state of the world, Cambridge University press. 515 pages.

Lovelock J. 1992, Gaia Comment soigner une Terre malade ? Editions Robert Laffont. 192 pages.

Monnier C. 2005, L'agriculture française en proie à l'écologisme, Moissons interdites. L'Hamattan. 238 pages 
Oudin B. 1996, Pour en finir avec les écolos. Editions Gallimard. 211 pages.

Prud'homme R. 2015, L'idéologie du réchauffement. Science molle et doctrine dure. L'artilleur. 283 pages.

Raoult D. 2016, Arrêtons d'avoir peur. Editions Michel Lafon, 348 pages.

Raoult D. 2015, De l'ignorance et de l'aveuglement: pour une science postmoderne. 265 pages

Raup D. M. 1993 De l'extinction des espèces; sur les causes de la disparition des dinosaures et de quelques milliards d'autres. Editions Gallimard NRF essais. 239 pages.

Revel J.-F. 1998 La connaissance inutile. Editions Grasset. 403 pages.

Revel J.F., 1997, Le voleur dans la maison vide. Editions Plon. 651 pages.

Revel J.-F., 2006, La grande parade, Essai sur la survie de l'utopie socialiste. Editions Plon. 345 pages.

Rittaud B. 2016, Ils s'imaginaient sauver le monde, Chroniques sceptiques de la COP21. Books éditions.214 pages.

de Rougemont M. 2017, Réarmer la raison; de l'écologie raisonnée à la politique raisonnable. MRint 272 pages

Sackett DL, Rosenberg WM, Gray JA, Haynes RB, Richardson WS. 1996, Evidence based medicine: what it is and what it isn't. BMJ. Jan 13;312(7023):71-72.
Schindler D.W., Hecky R. E., Findlay D. L., Stainton M. P., Parker B. R., Paterson M. J., Beaty M. K., Lyng M., Kasian S. E. M. 2008, Eutrophication of lakes cannot be controlled by reducing nitrogen input: Results of a 37-year whole-ecosystem experiment. PNAS August 12, vol. 105 no. 32: 11254- 11258 .

Schindler D.W., Carpenter S. R., Hecky R. E., Orihel D. M., 2016 Reducing Phosphorus to Curb Lake

Eutrophication is a Success, Environmental Science and Technology, 7p.

Simon J. 1998, The ultimate Ressource 2.

Stern N. 2006, Rapport Stern sur l'économie changement https://fr.wikipedia.org/wiki/Rapport_Stern climatique.

Schopenhauer A. 1833, L'Art d'avoir toujours raison, chapitre: "Stratagème $\mathrm{XXX} \quad$ - Argument d'autorité"https://fr.wikisource.org/wiki/L\%E2\%80\%99Art d\%E2\%80\%99avoir toujours raison Tirole J. 2016, Economie du bien commun. Editions PUF. 628 pages. 